\title{
Carotid artery stenosis
}

\section{An approach to its diagnosis and management}

Rohan Arasu, Alexis Arasu,

Juanita Muller

\section{Background}

Carotid artery stenosis (CAS) is one of the major causes of acute ischaemic stroke, accounting for approximately $20 \%$ of cases. It is not always symptomatic; however, when it is, the neurological vascular territory it commonly affects is the anterior circulation of the brain, causing symptoms such as hemiplegia, dysphasia or vision loss.

\section{Objective}

The aim of this article is to review the current literature on CAS, summarise the main updates and evidence base for surgical management, and discuss when vascular surgical input may be beneficial.

\section{Discussion}

CAS can be classified as symptomatic or asymptomatic disease. Carotid endarterectomy remains important in the treatment of symptomatic disease because of a strong evidence base for its benefit in the overall reduction of recurrent stroke risk. The benefit of surgery is less clear for asymptomatic disease. Commencement of best medical therapy as well as cardiovascular risk factor modification is a mainstay of treatment for both groups of patients.
CAROTID ARTERY STENOSIS (CAS) is a common condition encountered in general practice. It represents one of the major causes of acute ischaemic stroke, accounting for approximately $20 \%$ of cases. ${ }^{1}$ CAS can be classified as symptomatic or asymptomatic disease, with the distinction between the two significantly altering the management pathway. Acute neurological ischaemia or amaurosis fugax may be a manifestation of symptomatic disease, and patients presenting in this way should be promptly referred to an emergency department or discussed directly with a vascular surgical service. The other clinical scenario for CAS is asymptomatic disease that may be diagnosed following detection of a carotid bruit or as an incidental finding on imaging.

\section{Nature of the disease}

CAS is related to atherosclerotic plaque development in the internal carotid artery (ICA), which has a course both in the neck (extracranial) and within the skull (intracranial). Atherosclerosis itself is a chronic arterial disease related to the accumulation of cholesterol-lipid-calcium deposits in the walls of arteries. As it is typically a multisystem disease, patients with CAS will also be at risk of coronary artery disease or peripheral arterial disease and vice versa.

Neurological ischaemia can ensue following ICA plaque rupture and subsequent embolisation of atherosclerotic material that travels into the brain, causing arterial occlusion. ${ }^{2}$ Acute neurological ischaemia includes the syndromes of transient ischaemic attack (TIA) and stroke, which are described in Table 1 . The symptoms observed depend on the affected neurological vascular territories, as described in Table 2. The distribution of ischaemia and resulting symptoms from CAS are usually those from the anterior circulation (including the middle cerebral artery or anterior cerebral artery) or the retinal artery (which is an early branch of the ICA).

CAS usually does not cause neurological symptoms in the posterior circulation, as emboli from the ICA preferentially enter the anterior circulation as a result of the anatomy and the haemodynamics of the circle of Willis. ${ }^{2}$ CAS therefore is not typically considered a differential for symptoms such as vertigo or cerebellar dysfunction. Additionally, stenosis of the external carotid artery, which is an artery that supplies the face and neck, is usually benign and not considered to be a risk factor for stroke. 


\section{Symptomatic carotid artery stenosis} Symptomatic CAS relates to an episode of neurological ischaemia that has occurred in the preceding six months and has been linked to a haemodynamically significant ICA stenosis (ie stenosis $>50 \%$ ). There also needs to be appropriate exclusion of other causes. The symptoms are generally a sudden onset of sensory or motor impairment, dysphasia or mono-ocular vision loss (when the retinal artery is involved). It is important to note that visual symptoms will be ipsilateral to the CAS, while cerebral symptoms will be contralateral.

The natural history is that patients will frequently have either a single or multiple preceding TIA/s prior to a stroke. Unheralded strokes are relatively infrequent, ${ }^{3,4}$ in contrast to cardioembolic disease, which often causes a disabling stroke from the outset. ${ }^{5}$ Symptomatic carotid artery plaques are considered 'unstable' and more vulnerable to plaque rupture than asymptomatic plaques this pathophysiology is similar to acute coronary syndrome. A carotid artery will remain classified as symptomatic for a period of six months after the initial event. This time frame is linked to the period a symptomatic plaque has an elevated risk of recurrent stroke when compared with an asymptomatic plaque. ${ }^{6}$

Asymptomatic carotid artery stenosis Asymptomatic CAS is the presence of a haemodynamically significant CAS that has not caused an episode of neurological

Table 1. Neurological ischaemic syndromes ${ }^{23}$

\begin{tabular}{ll}
\hline Syndrome & Diagnostic criteria \\
\hline Transient ischaemic & Clinical criteria: ischaemic neurological symptoms that \\
attack (TIA) & resolve completely within 24 hours after onset \\
& Radiological criteria: presence of ischaemic neurological \\
& symptoms without evidence of infarction on central nervous \\
& system (CNS) imaging \\
\hline Stroke & Clinical criteria: ischaemic neurological symptoms persisting \\
& for a period of $>24$ hours \\
& Radiological criteria: presence of infarction on CNS imaging \\
\hline
\end{tabular}

ischaemia in the preceding six months. In most cases, asymptomatic disease is detected following auscultation of a carotid bruit, as an incidental finding on axial imaging or after carotid duplex ultrasonography (CDUS) evaluating symptomatic disease on the contralateral side.

\section{Imaging}

CDUS remains the first-line investigation for carotid artery disease, as it is an inexpensive, non-invasive modality that has been shown to be accurate in estimating the degree of stenosis when compared with angiographic measurement. ${ }^{7}$ The main drawback of the modality is that the accuracy of the test relies on the experience and ability of the sonographer. ${ }^{8}$ It should ideally be conducted at an accredited vascular laboratory, with the results classified according to the Australasian Society for Ultrasound in Medicine criteria. Computed tomography (CT) angiography can be a secondary imaging modality that is particularly useful for surgical planning.

\section{Management}

Management algorithms are best divided into three categories: symptomatic

Table 2. Vascular territories involved in neurological ischaemia with some of the commonly observed clinical symptoms and signs ${ }^{24}$

\begin{tabular}{|c|c|c|}
\hline Vascular territory & Commonly involved vessels & Common clinical symptoms and signs \\
\hline \multirow{4}{*}{ Anterior circulation } & \multirow{4}{*}{$\begin{array}{l}\text { Internal carotid artery } \\
\text { Anterior cerebral artery } \\
\text { Middle cerebral artery } \\
\text { Retinal artery }\end{array}$} & lower limb on the opposite side as the cerebral hemisphere involved \\
\hline & & $\begin{array}{l}\text { the tace, upper lımb and/or lower lımb on the opposite side as the } \\
\text { cerebral hemisphere involved }\end{array}$ \\
\hline & & $\begin{array}{l}\text { Dysphasia (if the dominant cerebral hemisphere is involved) - loss of the } \\
\text { ability to speak }\end{array}$ \\
\hline & & $\begin{array}{l}\text { Ipsilateral mono-ocular vision loss - complete loss of vision in a single } \\
\text { eye. This may be transient (amaurosis fugax) or permanent. }\end{array}$ \\
\hline \multirow[t]{3}{*}{ Posterior circulation } & Vertebral artery & \multirow{2}{*}{$\begin{array}{l}\text { Homonymous hemianopsia - visual field loss in either the right or left half } \\
\text { of both eyes }\end{array}$} \\
\hline & Basilar artery & \\
\hline & Cerebellar arteries & $\begin{array}{l}\text { Limb and gait ataxia - loss of coordination of the limbs and } \\
\text { uncoordinated walking }\end{array}$ \\
\hline
\end{tabular}


stenosis, asymptomatic stenosis and chronic occlusion.

\section{Management of symptomatic carotid artery stenosis}

All patients who present with acute neurological ischaemia or amaurosis fugax potentially related to symptomatic CAS should be referred urgently by either contacting a local vascular surgical service directly if the patient is known to have CAS, or referring the patient to the emergency department. The gold standard treatment for symptomatic disease is carotid endarterectomy (CEA), the main goal of surgery being to minimise the risk of recurrent stroke from an unstable plaque, with surgery having no impact on the outcome of the initial stroke. There are a number of landmark trials that have shown the benefit of CEA in symptomatic patients. ${ }^{9}$ Patients who underwent surgery and medical therapy were found to have a recurrent stroke risk of $9-15 \%$, which was significantly reduced when compared with a risk $>25 \%$ with medical therapy alone. ${ }^{4,10}$ For symptomatic patients with a stenosis of $\leq 49 \%$, the operation conferred no benefit. ${ }^{9}$

The timing of CEA after the neurological event is also critical, as the highest risk of recurrent stroke from a symptomatic ICA plaque is within the first two weeks from the initial event, with the risk remaining high for up to six weeks. ${ }^{6}$ This period is when the symptomatic plaque is considered to be most unstable with the highest risk of subsequent re-embolisation. Therefore, if surgery is being considered, it should ideally occur within two weeks from the initial event, a recommendation that is based on two large trials - the North American Symptomatic Carotid Endarterectomy Trial ${ }^{11}$ and the European Carotid Surgery Trial. ${ }^{10}$ Most vascular surgical services will work to facilitate surgery within the recommended two-week time frame, with patients often being admitted shortly after referral to ensure compliance to this time frame and for simultaneous care from stroke physicians.

Aside from referral to a vascular surgical service, treatment with antiplatelet therapy is also essential to reduce the risk of further neurological events from the unstable plaque. In general, advice should be sought from the vascular surgical service at the time of referral about the specific antiplatelet therapy that should be commenced. Importantly, antiplatelet therapy should be continued for the surgery and does not need to be withheld preoperatively.

\section{Management of asymptomatic carotid artery stenosis}

The commencement of best medical therapy (BMT) is the main principle of treatment for asymptomatic disease. This includes antiplatelet therapy, statin therapy and cardiovascular risk modification. The antiplatelet of choice for asymptomatic disease is generally aspirin monotherapy because of its overall low cost, good tolerability and equivalent efficacy when compared with other therapies. ${ }^{12,13}$ Other cardiovascular risk modifications are also imperative in CAS, including smoking cessation, blood pressure control, treatment of dyslipidaemia and screening for diabetes mellitus followed by optimisation of blood sugar control.

The role of surgery for asymptomatic disease continues to remain contentious in the vascular surgical community. The landmark studies supporting surgery for asymptomatic disease are the Asymptomatic Carotid Atherosclerotic Study ${ }^{14}$ and the Asymptomatic Carotid Surgery Trial, ${ }^{11}$ although the data used in these studies is more than 20 years old. Since then, the efficacy of medical management has significantly increased, especially when considering the significant improvements in the management of hypertension, dyslipidaemia and diabetes mellitus. Therefore, medical therapy alone is now considered by many to be increasingly comparable to surgical management for stroke risk prevention in asymptomatic disease. ${ }^{15}$ Given these factors and the lack of modern randomised controlled trials comparing medical therapy to surgery, there is a diversity of opinion and practice.

A reasonable approach from a general practice point of view is to refer asymptomatic patients with an
ICA stenosis of $>80 \%$ for a non-urgent vascular surgical review for consideration of surgery. ${ }^{16}$ The likely scenarios that could occur would be either the decision for medical management only with ongoing surveillance or the decision for surgery after careful consideration of the cumulative stroke reduction risk in that particular patient. Generally, patients who benefit most from surgery are those who are young and have few comorbidities, as these patients will accumulate the most stroke risk reduction over their lifetimes. For asymptomatic patients with an ICA stenosis of $<80 \%$, referral is usually not required. These patients should have annual surveillance with CDUS to monitor the progression of stenosis. In general, older patients benefit less from surgery as they will have an overall lower cumulative stroke reduction risk from surgery while also having a higher potential complication rate. Some consideration could therefore be given to stopping surveillance in elderly patients, especially in those with significant comorbidities. ${ }^{11}$

\section{Management of chronic carotid artery occlusion}

Chronic carotid artery occlusion in patients without acute neurological ischaemic symptoms is generally a benign condition. The overall risk of stroke from the vessel is mostly mitigated, as emboli cannot be carried into the intracranial circulation without blood flow through the vessel. ${ }^{17}$ These patients should still commence BMT as they remain at risk for other forms of atherosclerotic disease; however, they generally do not need referral to a vascular surgeon as these patients do not benefit from surgery. ${ }^{17}$

\section{Carotid endarterectomy}

CEA is an operation that involves open exposure of the carotid artery followed by removal of atherosclerotic plaque and reconstruction of the artery. The most significant complication is perioperative stroke, occurring at a rate of just above $2 \% .{ }^{18}$ The other relevant complication is injury to cranial nerves in the region, particularly the hypoglossal nerve, glossopharyngeal nerve and vagus nerve. 
Most cranial nerve deficits are transient, with the risk of permanent injury being relatively low, occurring in approximately $0.5 \%$ of patients. ${ }^{19}$

Follow-up after CEA usually involves CDUS at approximately the 4-6-week mark and another at the six-month mark. These scans are used to assess for restenosis of the carotid artery. If these early scans are normal, the risk of developing late restenosis is low. ${ }^{20}$

\section{Carotid artery stenting}

Carotid artery stenting is the endovascular insertion of a covered stent to exclude the diseased portion of the ICA from the circulation. It is currently not the preferred technique as it has a significantly increased rate of perioperative stroke when compared with that of CEA. ${ }^{21,22}$ It has utility in situations where CEA is contraindicated, such as in those patients with a prohibitive general anaesthetic risk or those with a 'hostile neck' from previous surgery or radiotherapy to that region.

\section{Conclusion}

Carotid artery disease is a complex topic, with management differing significantly based on whether the disease is symptomatic or asymptomatic. The predominant demographic of patients that general practitioners will be involved in managing will be those who are asymptomatic, and it is increasingly felt that medical management may be comparable in efficacy to surgical management. Additionally, many patients with CAS will have other forms of atherosclerotic disease, and commencement of BMT will help slow the progression of these other life-threatening diseases as well.

\section{Key points}

- CAS is a major cause of acute ischaemic stroke.

- The disease can be classified as asymptomatic or symptomatic disease on the basis of the correlation of the disease with a recent stroke or TIA.

- Patients presenting with acute neurological ischaemia or amaurosis fugax, which may be a manifestation of symptomatic CAS, should be promptly referred to an emergency department or discussed directly with a vascular surgical service.

- Patients with symptomatic CAS should be commenced on antiplatelet therapy and considered for surgery.

- Patients with asymptomatic CAS should be commenced on BMT with referral to a vascular surgical service when the degree of stenosis exceeds $80 \%$. If the degree of stenosis is $<80 \%$, yearly surveillance with CDUS is appropriate.

- CEA is the gold standard for the surgical management of symptomatic CAS and should ideally be carried out within two weeks from the initial event. It aims to reduce the recurrent stroke risk with no impact on the outcome of the initial stroke.

\section{Authors}

Rohan Arasu MBBS, GDipSurgAnat, Principal House Officer, Department of Vascular Surgery, Princess Alexandra Hospital, Woolloongabba, Qld

Alexis Arasu MBBS (Hons), BMedSc (Hons), Medical Intern, Monash Health, Melbourne, Vic Juanita Muller MBBS, FRACS (Vasc), Vascular Surgeon, Department of Vascular Surgery, Princess Alexandra Hospital, Woolloongabba, Qld Competing interests: None.

Funding: None.

Provenance and peer review: Not commissioned, externally peer reviewed.

Correspondence to:

rohan_arasu@outlook.com

\section{References}

1. Benjamin EJ, Virani SS, Callaway CW, et al. Heart disease and stroke statistics - 2018 update: A report from the American Heart Association Circulation 2018;137(12):e67-492. doi: 10.1161/ CIR.0000000000000558.

2. Golledge J, Greenhalgh RM, Davies AH. The symptomatic carotid plaque. Stroke 2000;31(3):774-81. doi: 10.1161/01.str.31.3.774.

3. Dodick DW, Meissner I, Meyer FB, Cloft HJ. Evaluation and management of asymptomatic carotid artery stenosis. Mayo Clin Proc 2004;79(7):937-44. doi: 10.4065/79.7.937.

4. North American Symptomatic Carotid Endarterectomy Trial Collaborators, Barnett HJM, Taylor DW, et al. Beneficial effect of carotid endarterectomy in symptomatic patients with high-grade carotid stenosis. N Engl J Med 1991;325(7):445-53. doi: 10.1056/ NEJM199108153250701.

5. Arboix A, Oliveres M, Massons J, Pujades $\mathrm{R}$, Garcia-Eroles L. Early differentiation of cardioembolic from atherothrombotic cerebral infarction: A multivariate analysis. Eur J Neurol 1999;6(6):677-83. doi: 10.1046/j.14681331.1999.660677.x
6. Rothwell PM, Eliasziw M, Gutnikov SA, Warlow CP, Barnett HJ, Carotid Endarterectomy Trialists Collaboration. Endarterectomy for symptomatic carotid stenosis in relation to clinical subgroups and timing of surgery. Lancet 2004;363(9413):915-24. doi: 10.1016/S01406736(04)15785-1.

7. Wardlaw JM, Chappell FM, Best JJ, Wartolowska K, Berry E, NHS Research and Development Health Technology Assessment Carotid Stenosis Imaging Group. Non-invasive imaging compared with intra-arterial angiography in the diagnosis of symptomatic carotid stenosis: A meta-analysis. Lancet 2006;367(9521):1503-12. doi: 10.1016/S0140-6736(06)68650-9.

8. Criswell BK, Langsfeld M, Tullis MJ, Marek J. Evaluating institutional variability of duplex scanning in the detection of carotid artery stenosis. Am J Surg 1998;176(6):591-97. doi: 10.1016/s0002-9610(98)00287-6.

9. Rothwell PM, Eliasziw M, Gutnikov SA, et al. Analysis of pooled data from the randomised controlled trials of endarterectomy for symptomatic carotid stenosis. Lancet 2003;361(9352):107-16. doi: 10.1016/s01406736(03)12228-3.

10. Group ECSTC. Randomised trial of endarterectomy for recently symptomatic carotid stenosis: Final results of the MRC European Carotid Surgery Trial (ECST). Lancet 1996;351(9113):1235-43.

11. Halliday A, Harrison M, Hayter E, et al. 10-year stroke prevention after successful carotid endarterectomy for asymptomatic stenosis (ACST-1): A multicentre randomised trial. Lancet 2010;376(9746):1074-84. doi: 10.1016/S01406736(10)61197-X

12. Diener HC, Bogousslavsky J, Brass LM, et al. Aspirin and clopidogrel compared with clopidogrel alone after recent ischaemic stroke or transient ischaemic attack in high-risk patients (MATCH) Randomised, double-blind, placebo-controlled trial. Lancet 2004;364(9431):331-37. doi: 10.1016/ S0140-6736(04)16721-4.

13. CAPRIE Steering Committee. A randomised, blinded, trial of clopidogrel versus aspirin in patients at risk of ischaemic events (CAPRIE). Lancet 1996;348(9038):1329-39. doi: 10.1016/ s0140-6736(96)09457-3. PMID: 8918275.

14. Rothwell PM, Goldstein LB. Carotid endarterectomy for asymptomatic carotid stenosis: Asymptomatic carotid surgery trial. Stroke 2004;35(10):2425-27. doi: 10.1161/01. STR.0000141706.50170.a7.

15. Abbott AL. Medical (nonsurgical) intervention alone is now best for prevention of stroke associated with asymptomatic severe carotid stenosis: Results of a systematic review and analysis. Stroke 2009;40(10):e573-83. doi: 10.1161/ STROKEAHA.109.556068.

16. Naylor AR, Ricco JB, de Borst GJ, et al. Editor's choice - Management of atherosclerotic carotid and vertebral artery disease: 2017 clinical practice guidelines of the European Society for Vascular Surgery (ESVS). Eur J Vasc Endovasc Surg 2018;55(1):3-81. doi: 10.1016/j.ejvs.2017.06.021.

17. Hackam DG. Prognosis of asymptomatic carotid artery occlusion: Systematic review and metaanalysis. Stroke 2016;47(5):1253-57. doi: 10.1161/ STROKEAHA.116.012760.

18. Reddy RP, Karnati T, Massa RE, Thirumala PD. Association between perioperative stroke and 30-day mortality in carotid endarterectomy: A meta-analysis. Clin Neurol Neurosurg 2019;181:44-51. doi: 10.1016/j. clineuro.2019.03.028. 
19. Greenstein AJ, Chassin MR, Wang J, et al. Association between minor and major surgical complications after carotid endarterectomy: Results of the New York Carotid Artery Surgery study. J Vasc Surg 2007;46(6):1138-44; discussion 1145-46. doi: 10.1016/j.jvs.2007.08.026.

20. Kallmayer M, Tsantilas P, Zieger C, et al. Ultrasound surveillance after CAS and CEA: What's the evidence? J Cardiovasc Surg (Torino) 2014;55(2 Suppl 1):33-41. PMID: 24796896.

21. LaMuraglia GM, Brewster DC, Moncure AC, et al. Carotid endarterectomy at the millennium: What interventional therapy must match. Ann Surg 2004;240(3):535-44; discussion 544-46. doi: 10.1097/01.sla.0000137142.26925.3c.
22. Brott TG, Hobson RW 2nd, Howard G, et al. Stenting versus endarterectomy for treatment of carotid-artery stenosis. N Engl J Med 2010;363(1):11-23. doi: 10.1056/NEJMoa0912321.

23. Sacco RL, Kasner SE, Broderick JP, et al. An updated definition of stroke for the 21st century: A statement for healthcare professionals from the American Heart Association/American Stroke Association. Stroke 2013;44(7):2064-89. doi: 10.1161/STR.0b013e318296aeca.

24. Tao WD, Liu M, Fisher M, et al. Posterior versus anterior circulation infarction: How different are the neurological deficits?

Stroke 2012;43(8):2060-65. doi: 10.1161/ STROKEAHA.112.652420. 